\title{
SP-SEM Observation of Magnetic Vortex States in Permalloy Disks
}

\author{
Y. Yamada, M. Nakamura, K. Sueoka, and K. Mukasa \\ Graduate School of Engineering, Hokkaido University, Kita-13, Nishi-8, Kita ku, Sapporo 060-8628
}

\begin{abstract}
The magnetic vortex in a ferromagnetic disk has a unique feature of spin configuration to eliminate magnetostatic energy with a vertical bistable magnetization characteristics. We have observed magnetic vortex states in permalloy thin film disks with diameters ranging from $2 \mu \mathrm{m}$ to $25 \mu \mathrm{m}$, using spin-polarized scanning electron microscopy (SP-SEM). Magnetic vortex states exist throughout range of diameters. At diameters larger than $15 \mu \mathrm{m}$, multidomain states were also observed, however, a magnetic vortex state should be expected as one of the low-energy states even in a ferromagnetic disk as large as $25 \mu \mathrm{m}$.
\end{abstract}

Key words: magnetic vortex, SP-SEM

\section{Introduction}

To develop novel high-density magnetic data storage systems, such as magnetoresistive memories and spin-controlled logic devices, it has been important to investigate the magnetic domain structure and its dynamics in nanoscale magnetic elements. A ferromagnetic circular disk is a typical candidate for a nanomagnet, and its magnetic features have been discussed 1). Recently the circular nanomagnet has attracted much attention after the report of a demonstration experiment in which a single spin vortex state was observed in the disk by means of a magnetic force microscope (MFM) ${ }^{2)}$. The magnetic vortex in a ferromagnetic disk has a unique feature of spin configuration to eliminate magnetostatic energy with vertical bistable magnetization characteristics ${ }^{3), 4)}$. Dynamic processes to create or annihilate vortex cores (vertical spin component at the center of the disk) ${ }^{5), 6), 7)}$ and stability of magnetic vortices have been discussed in the past few years ${ }^{8), 9)}$. In these experimental reports, two kinds of magnetic imaging techniques have been used. One is MFM, which gives a clear image of vortex cores, sometimes accompanied by weak magnetic contrast associated with imperfection of the closure spin configuration. The other is Lorentz transmission electron microscopy (LTEM), which records deflection of electron beams by circular magnetic field inside the disk. LTEM is a method complementary to MFM because MFM detects the leakage magnetic field vertically from the disk surface and LTEM is sensitive only to the in-plane magnetic field component.

Other magnetic imaging techniques are also applicable to investigation of vortex states. Electron holography has given the interference patterns induced by a circular in-plane distribution of magnetization in thin plates of $\mathrm{Co}^{\left.- \text {crystal }^{10}\right)}$. Spin-polarized scanning electron microscopy (SP-SEM, called Spin-SEM) ${ }^{11)}$ and scanning electron microscopy with polarization analysis (SEMPA) are potential techniques resolving the in-plane component of spins that enable one to distinguish the chirality of the vortex, as well as the vertical component associated with the direction of the vortex core. In this paper, we report SP-SEM observation of a magnetic vortex. In our earlier work on analytical investigation of the magnetic vortex state ${ }^{3)}$ without circumferential boundary conditions, an assumption of cylindrical symmetry of the system was enough to prove that the magnetic vortex state is a stable state with a magnetic energy minimum. If the disk is perfectly fabricated, the disk size for formimg a curling magnetization structure might be unlimited. There is no report on MFM or LTEM observation of a disk with a diameter larger than a few micrometers; therefore, we have observed surface spin states of permalloy disks of 2 to $25 \mu \mathrm{m}$ in diameter.

\section{Experiment}

The SP-SEM used in this study was developed in our laboratory $\left.{ }^{12)}, 13\right)$. The electron spin analyzer used in the SP-SEM is a compact retarding-type Mott polarimeter that we designed. Originally the polarimeter had two pairs of scattering electron detectors, but only one pair was available for this experiment. Fig. 1 shows the geometric relations of primary electrons, secondary electrons, sample surface, spin-sensitive direction of the system. During SP-SEM measurement, pressure in the sample chamber was maintained below $1 \times 10^{-9}$ Torr. The primary electrons accelerate to $20 \mathrm{keV}$ with a probe current of $10 \mathrm{nA}$ focused into a spot of about $60 \mathrm{~nm}$ in diameter. Spin-polarized secondary electrons emanating from the sample surface were collected by a static electric field of about $200 \mathrm{eV}$ and transferred to the polarimeter. To reduce stray magnetic fields from a magnetic objective lens, we designed a magnetic shield attached over the pole piece. The target gold film in the

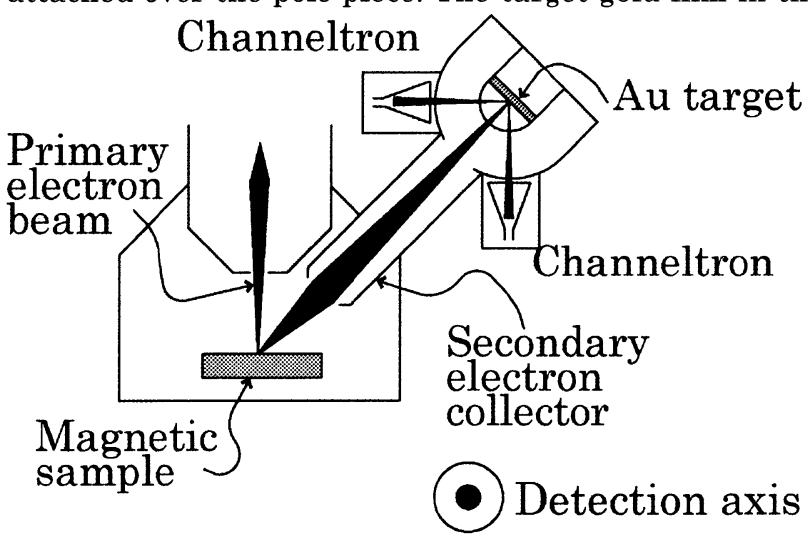

Fig. 1 Schematic diagram of SP-SEM. Spin-polarized secondary electrons were collected by a static electric field of about $200 \mathrm{eV}$ and transferred to the polarimeter. The asymmetry was determined by counting the scattered electrons with two channeltrons located on opposite sides at $-120^{\circ}$ and $+120^{\circ}$ scattering directions. 
polarimeter was maintained at $32.5 \mathrm{kV}$ for spin-dependent Coulomb scattering. The polarimeter was calibrated by measuring the spin polarization of the secondary electrons observed on a clean (001) surface of an Fe single crystal.

The ferromagnetic disks were made of permalloy thin films, using conventional electron beam lithography, magnetron sputtering deposition, a liftoff technique. After fabricating the thin-film disks on a Si substrate, we transferred the substrate into the SP-SEM chamber through the air. Prior to SP-SEM observation, surface cleaning by an $\mathrm{Ar}^{+}$ion-sputtering gun was necessary to remove an oxidized layer and an adsorbed layer which reduce spin polarization of secondary electrons. We observed permalloy disks of $2,4,6,8,10,12,15,20$, and $25 \mu \mathrm{m}$ in diameter, all of which were $50 \mathrm{~nm}$ in thickness.

SEM and spin images of a permalloy disk $10 \mu \mathrm{m}$ in diameter are shown in Fig. 2. The spin images map the asymmetry of scattered electrons detected by two electron counters located on the left and right sides as shown in Fig. 1. The SEM images are constructed by summing the number of scattered electrons. Fig. 2(a) is the SEM image and Figs. 2(b) and 2(c) are the spin image. Since only one pair of electron counters was used in this experiment, the contrast of the spin image corresponds to the spin component along the direction perpendicular to the line connecting the two detectors. We define this direction as the $x$ direction. Spin components parallel to the $x$ direction are imaged as bright dots, and the area of negative spin-polarization components along this direction is painted as a dark region. To observe the spin-polarization components along the $y$ direction, the sample was rotated 90 degrees. Fig. 2(c) shows the $y$ component of spin-polarization of secondary electrons. These spin images suggest that spins on the disk surface aligned along the circumferential direction and the spins curl in the counterclockwise direction.

The signal-to-noise ratio of the spin images depends

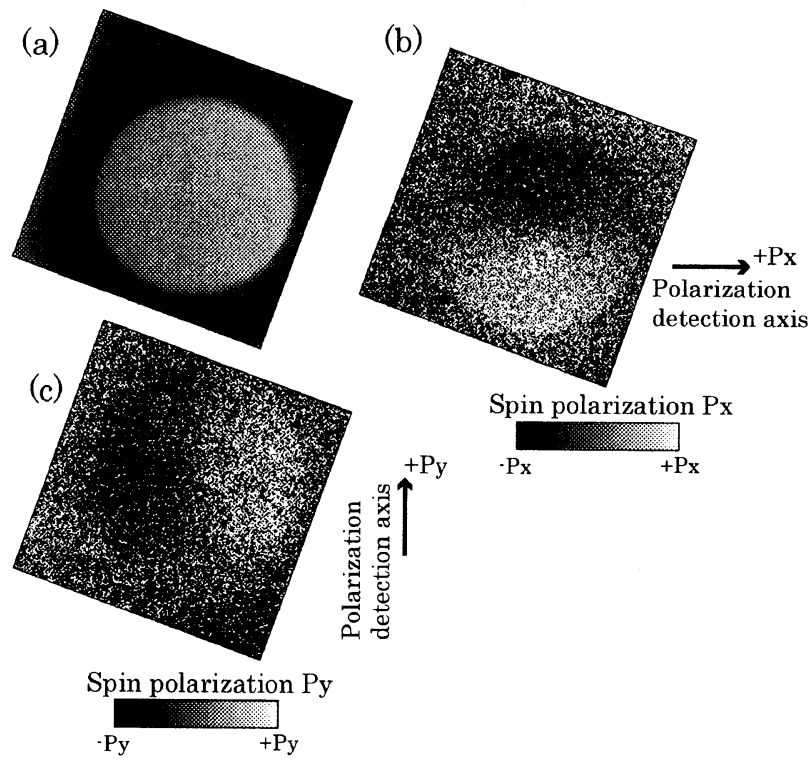

Fig. 2 (a) SEM image, (b) spin image of $x$ direction, and (c) spin image of $y$ direction. These images indicate that the magnetization vector rotates gradually along the edge of a circular disk.

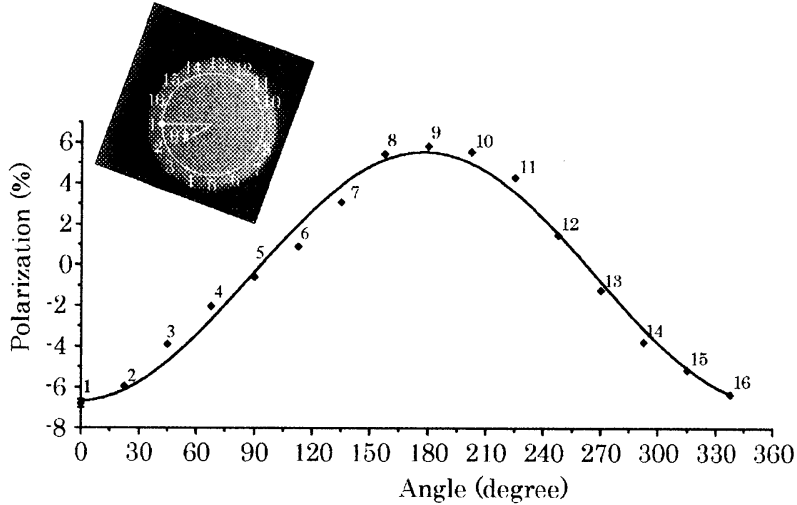

Fig. 3 Spin-polarization in a circular disk. Sixteen individual measurement points were selected as shown in the inset. The dotted line is the cosine fit of the data. Small deviation of the dots indicates that the magnetization rotates coherently along the circumference. It took about $50 \mathrm{~s}$. to measure the spin-polarization at each point.

upon the acquisition time to obtain the images, because of low efficiency; $I / I_{0}<1 \times 10^{-4}$ ( $I$ is the detected total electron current, $I_{O}$ is secondary electron current ). To define the spin-polarization more clearly, spin-polarizations of one pixel are measured with an acquisition time about one thousand times as long as the case shown in Figs. 2(b) and 2(c). Dots in Fig. 3 show the spin-polarization measured at 16 individual points on an $8 \cdot \mu \mathrm{m}$ circle inside the disk, as shown in an inset of Fig. 3. These measurements were made on the same disk as Fig. 2. Because of the large acquisition time per point, the statistical error is too small in Fig. 3. The dotted line is a fitted cosine curve. Small deviation of the dots indicates that the magnetization rotates coherently along the circumference. The observed

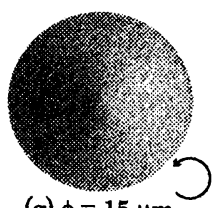

(g) $\phi=15 \mu \mathrm{m}$
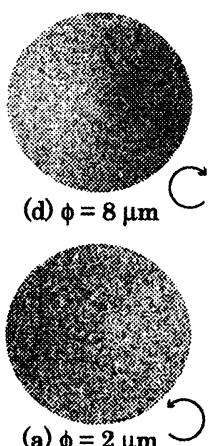

(a) $\phi=2 \mu \mathrm{m}$

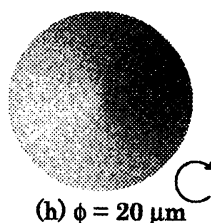

(h) $\phi=20 \mu \mathrm{m}$

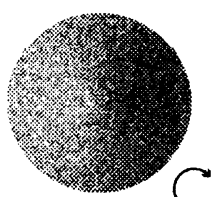

(e) $\phi=10 \mu \mathrm{m}$

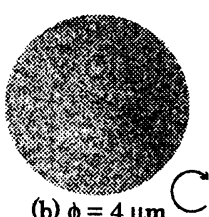

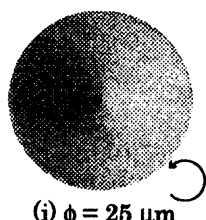
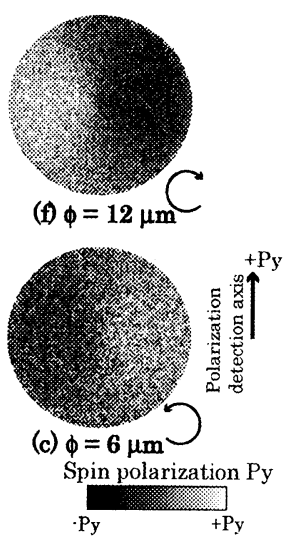

Fig. 4 Spin images of permalloy disks $2,4,6,8,10$, $12,15,20$, and $25 \mu \mathrm{m}$ in diameter, all being $50 \mathrm{~nm}$ in thickness. Images 4(a), 4(c), 4(g), and 4(i) indicate spin curling counterclockwise, and $4(\mathrm{~b}), 4(\mathrm{~d}), 4(\mathrm{e})$, $4(\mathrm{f})$, and $4(\mathrm{~h})$ indicate clockwise curling. 
maximum spin-polarization is about $6 \%$. We confirmed that this value coincides with the spin-polarization measured on the magnetic domain parallel to the observing direction, using a closure domain structure emerging on a rectangular permalloy thin film. Small deviations of measured points from the fitted curve were caused by errors in the measured position.

Fig. 4 shows typical spin images observed for each disk size. These are the as-grown states of the magnetic vortex, and their chirality was randomly observed. Figs. 4(a), 4(c), 4(g), and 4(i) are the magnetic vortices with spins curling counterclockwise; the contrast reversal in Figs. 4(b), 4(d), 4(e), 4(f), and 4(h) means the curling is clockwise. The smaller the diameter of the disks, the more the spin contrast blurred because of electron beam induced contamination. Contaminants reduce the spin-polarization of secondary emitted electrons. Since the beam scan time over the disks is almost the same for all disk diameters, this effect is dominant for disks of small diameter.

We observed a few samples at each diameter, and almost all samples showed magnetic vortex states. However, one exception was observed in the samples larger than $15 \mu \mathrm{m}$. This indicates that the vortex state is a stable state ${ }^{4}$. Fig. 5(a) shows an SP-SEM image of the exception, with a multidomain state observed on a disk $15 \mu \mathrm{m}$ in diameter (as-grown state). After the SP-SEM observation, we observed the same sample by MFM as shown in Fig. 5(b). It can be concluded that the same domain structure was observed by two different magnetic imaging methods. To change the multidomain structure into the vortex state, a magnetic field of $8 \mathrm{kOe}$

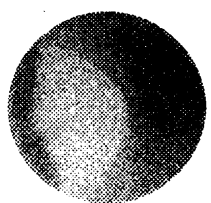

(a) Spin image

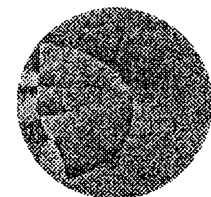

(b) MFM image

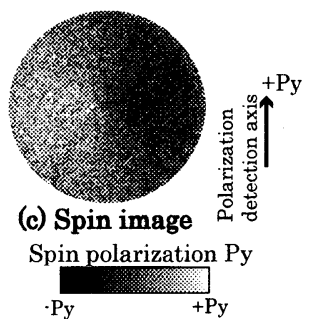

$\cdot \mathrm{Py}$
Fig. 5 (a) Spin image of a circular permalloy disk 15 $\mu \mathrm{m}$ in diameter (as-grown state), (b) MFM image of $5(\mathrm{a})$, and (c) spin image of 5(a) after a magnetic field of $8 \mathrm{kOe}$ was applied perpendicular to the film.

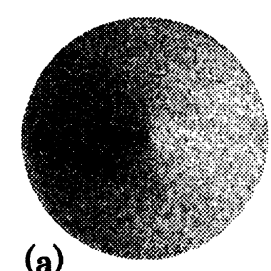

(a)

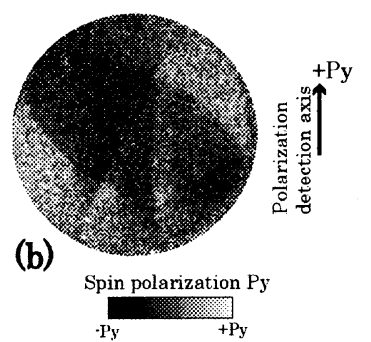

Py
Fig. 6 (a) Spin image of a circular permalloy disk 25 $\mu \mathrm{m}$ in diameter (as-grown state) shows a magnetic vortex state. (b) After a magnetic field of $8 \mathrm{kOe}$ was applied perpendicular to the film, the magnetic vortex state changed to a multidomain state. was applied perpendicular to the film. Fig. 5(c) indicates that the magnetic vortex state was recovered.

With the $25-\mu \mathrm{m}$ disk, the opposite situation was observed. The magnetic vortex state observed as an initial state, as shown in Fig. 6(a), changed into a multidomain state, as shown in Fig. 6(b), after the application of $8 \mathrm{kOe}$ perpendicular to the film. This situation was often observed. According to the perpendicular magnetization curve of a $1 \mu \mathrm{m}$ permalloy disk reported by Kikuchi et al. ${ }^{4)}$, the multidomain structure is not expected to be observed as a remanent state after application of perpendicular magnetic fields. Due to the difference in experimental conditions, such as an inhomogeneity of the film in large disks, there is a possibility that the multidomain state is one state at an energy local minimum. Occasionally we observed that the multidomain structure changed into the vortex state during the MFM measurement. The multidomain structure was not stable and was easily perturbed by a magnetic field. To clear these arguments, numerical simulations should be performed to obtain the magnetic energy and stable domain states.

\section{Conclusion}

We observed magnetic vortex states in permalloy thin film disks with diameters ranging from $2 \mu \mathrm{m}$ to $25 \mu \mathrm{m}$, using a spin-polarized scanning electron microscope. Magnetic vortex states exist at all these diameters. At the diameters larger than $15 \mu \mathrm{m}$, multidomain states were also observed, but the magnetic vortex state should be expected as a low-energy state even in the $25-\mu \mathrm{m}$ ferromagnetic disk. To discuss the stability of the vortex states and the limitation of the diameter that permits existence of the vortex, statistical analysis of the states with several samples is required. In contrast to the MFM measurement, the in-plane component of magnetic moment can be resolved by SP-SEM without magnetic perturbations caused by magnetic interactions between the sample and the probes. SP-SEM can image complex multidomain structures that cannot be distinguished by the LTEM method. Due to large spot size of the primary electron beam (about $60 \mathrm{~nm}$ ), thermal drift of the sample stage, and poor vibration stability of our system, the vortex core was not resolved. These mechanical problems can be solved, and vortex cores are to be imaged with our instrument in the future.

\section{References}

1) R. Cowburn, D. Koltsov, A. Adeyeye, and M. Walland, Phys. Rev. Lett., 83, 1042(1999).

2) T. Shinjo, T. Okuno, R. Hassdorf. K. Shigeto, and T. Ono, Science 289, 930 (2000).

3) E. Hirota, R. Nakane, M. Sawamura, S. Chikazumi, and K. Mukasa, J. Mag. Soc. Jpn., 23, 599 (1999).

4) N. Kikuchi, S. Okamoto, O. Kitakami, Y. Shimada, S. Kim, Y. Otani, and K. Fukamichi, J. Appl. Phys., 90, 6548 (2001).

5) T. Pokhi, D. Song, and J. Nowak, J. Appl. Phys., 87, 6319 (2000).

6) T. Okuno, K. Sigeto, T. Ono, K. Mibu, and T. Shinjo, J. Magn. Magn. Mat., 240, 1 (2000). 
7) M. Schnider, H. Hoffmann, and J. Zweck, Appl. Phys. Lett., 78, 3113 (2001).

8) M. Schneider, H. Hoffmann, S. Otto, Th. Haug, and J. Zweck, J. Appl. Phys., 92, 1466 (2002).

9) K. Metlov and K. Guslienko, J. Magn. Magn. Mat., 242-245, 1015 (2002).

10)A. Tonomura, M. Matsuda, J. Endo, T. Arai, and K. Mishima. Phys. Rev. Lett., 44, 1430 (1980).

11)K. Koike and K. Hayakawa, J. Appl. Phys. 57, 4244(1985).

12)Y. Kuraoka, K. Sueoka, T. Iwata, K. Mukasa, R. Aihara, and K. Hayakawa, J. Magn. Soc. Jpn., 23, 727 (1999).

13)Y. Kuraoka, K. Sueoka, T. Iwata, K. Mukasa, R. Aihara, and K. Hayakawa, J. Magn. Soc. Jpn., 22, 389 (1998).

Received Oct. 7, 2002; Accepted Jan. 17, 2003 